\title{
Two-way relaying schemes in full duplex cellular system
}

\author{
Yabo Shi, Meng Ma* (D), Chang Liu and Bingli Jiao
}

\begin{abstract}
Recently, full duplex (FD) has been attracting great attention, due to its capability to double the spectral efficiency. In this paper, we focus on a FD wireless communication network, in which a FD base station (BS) and a half duplex (HD) mobile station (MS) exchange their information via a HD relay station (RS) within two phases. An amplify-and-forward (AF) relaying scheme and a decode-and-forward (DF) relaying scheme are proposed for the two-way relay network. In the proposed DF relaying scheme, the RS chooses the best DF relaying mode according to channel state information (CSI) to achieve the maximum capacity. Specifically, the RS can decode and forward data streams from both the BS and MS, or only one of them, or none of them, and thus obtaining a selection diversity gain. In order to analyze the performance of the proposed schemes, achievable rate regions and sum-capacities of the proposed schemes are derived in closed form. Numerical and simulation results show that the proposed relaying schemes provide significant capacity gain.
\end{abstract}

Keywords: Achievable rate regions, Full duplex radios, Scheme design, Relaying mode selection, Capacity maximization

\section{Introduction}

Traditionally, wireless communication nodes operate in half duplex (HD) mode, i.e., in either time division duplex (TDD) mode or frequency division duplex (FDD) mode. Recently, a new duplex technique, which called cofrequency co-time full duplex (FD), has been attracting great attention, due to its potential capability to double the spectral efficiency [1-4]. In FD mode, the interference from transmitter to its own receiver should be canceled effectively. In the past few years, a great progress has been made on the self-interference (SI) cancelation issue $[3,5,6]$. By jointly employing antenna isolation, radio frequency cancelation, and digital cancelation, the interference cancelation ability can achieve more than $110 \mathrm{~dB}$ [2]. The SI can be reduced to much lower than the desired signal by using the methods proposed in [5-7], thus making the FD technique possible in some application scenarios. Many studies investigating the performance of FD communication either assume perfect self-interference cancelation [8] or treat the residual interference as a Gaussian noise [9].

*Correspondence: mam@pku.edu.cn

School of Electronic Engineering and Computer Science, Peking University, Beijing 100871, China
One of the important application scenarios of FD is relay networks $[10,11]$. As relays can enhance coverage, throughput, and reliability of wireless communication systems, it has attracted intense research interest in the past decades [12-19]. Performance analyses show that the FD relay system outperforms the traditional HD relay systems [20-24].

In relay networks, there are two basic relaying schemes, i.e., amplify-and-forward (AF) relaying scheme and decode-and-forward (DF) relaying scheme [25]. In the former scheme, the relay amplifies the received signal from the source and forwards it to the destination [17]. In the latter scheme, the relay first decodes the received signal and then re-encodes and forwards it to the destination [26, 27].

In designing the relaying schemes for FD relay networks, one should consider not only the forward scheme, but also the duplex mode of each node. In fact, the FD technique enables the relay network operates in a hybrid duplex mode, i.e., some nodes work in FD mode and the other nodes work in HD mode. In a two-way relay network, which consists of two source nodes exchanging information with the assistance of relay stations (RSs), according 
to the duplex mode of resource nodes and RSs, the FD relaying network can be divided into four kinds. The first one is a relay system consisting of one FD RS and two HD source nodes. This kind of system has been extensively studied in the last decade [28-31]. For example, two hybrid relaying schemes opportunistically switching between FD and HD relaying modes are proposed in [29] to maximize the instantaneous spectral efficiency, and in [31] to minimize the outage probability, respectively. The second kind is a relay system consisting of a FD RS and two FD source nodes. This kind of system is first studied in [32], where achievable rate regions are derived for different relaying schemes, including DF, compress-andforward (CF), and AF relaying schemes. In [33], a relaying system with two FD source nodes and multiple FD RSs is studied, and a relay selection method is proposed for AF relaying scheme to achieve the maximum signal-tointerference-plus-noise ratio (SINR). The third kind is the relay network consisting of one FD RS, HD source nodes, and FD source nodes, which has been insufficiently studied so far. In [23], a system consisting of a FD RS and two HD source nodes is studied, and the advantages and disadvantages are analyzed in terms of sum-rate and resource wastage. In [34], a hybrid-scheduling algorithm is proposed for the third kind of system based on a three-step relaying scheme. The fourth kind of FD relay network is a relay system consisting of one HD RS and two source nodes, in which at least one source node works in FD mode. This kind of system can be widely used in cooperative communication scenarios. For example, in Internet of things (IoT) networks and vehicular communication networks [35], the devices or terminals can establish an ad hoc network, and each of them can play a role as a RS to assist another devices or terminals to communicate with a base station (BS) or an access point. To the best of the authors' knowledge, this kind of system has not been studied yet.

The contribution of this paper is threefold. First, an AF relaying scheme is proposed, and sum-capacity is derived in closed form. Second, a hybrid DF relaying scheme is proposed, in which the RS chooses the best DF relaying mode according to channel state information (CSI) to achieve the maximum capacity. Specifically, the RS can decode and forward data streams from both the BS and mobile station (MS), or only one of them, or none of them, and thus obtaining a selection diversity gain. Moreover, the achievable rate regions and sum-capacities of each relaying mode are derived in closed form. Third, the proposed DF relaying scheme are analyzed for different channel conditions. Theoretical analysis and simulation results not only substantiate the significant capacity gains obtained by the proposed DF relaying scheme but also provide useful tools for best relaying mode selection in cellular systems.
The rest of this paper is organized as follows. In Section 2, a system model and assumptions are described. An AF relaying scheme is proposed and analyzed in Section 3. In Section 4, DF relaying scheme based on four kinds of relaying modes is proposed, and the achievable rate region and sum-capacity of the proposed DF relaying scheme is also presented. In Section 5, performances of the proposed AF and DF relaying schemes are evaluated by simulations. Finally, conclusions are drawn in Section 6 .

\section{System model}

Let us consider a bidirectional relay system, which consists of one BS working in FD mode, and one MS and one RS working in HD-TDD mode. A bidirectional link between BS and MS is established with cooperation of RS. As both the RS and MS operate in HD mode, it requires, at least, two phases for information exchange between the BS and MS, as shown in Fig. 1. At the first phase, the BS and MS transmit their signals to RS, and meanwhile, the $\mathrm{BS}$ also receives the uplink signal from MS, since it works in FD mode. Thus, the RS receives a mixed signal. At the second phase, in the AF relaying scheme, the RS amplifies and forwards the received signal to the BS and MS, while in the FD scheme, the RS can decode and forward the data streams from both the BS and MS, or only one of them, or none of them, according to the channel state information (CSI). Meanwhile, the BS also transmits the downlink signal to the MS. As a result, the MS receives a combination of the two signals, one from the RS and the other from the BS simultaneously.

In this paper, the proposed AF and DF relaying schemes are all based on the above-mentioned two-phase manner. The transmission and reception strategies at the first phase are all the same for the proposed schemes. The main problem we are addressing here is to design the transmission strategy at the second phase, especially the forwarding mode at the RS. Before presenting the proposed

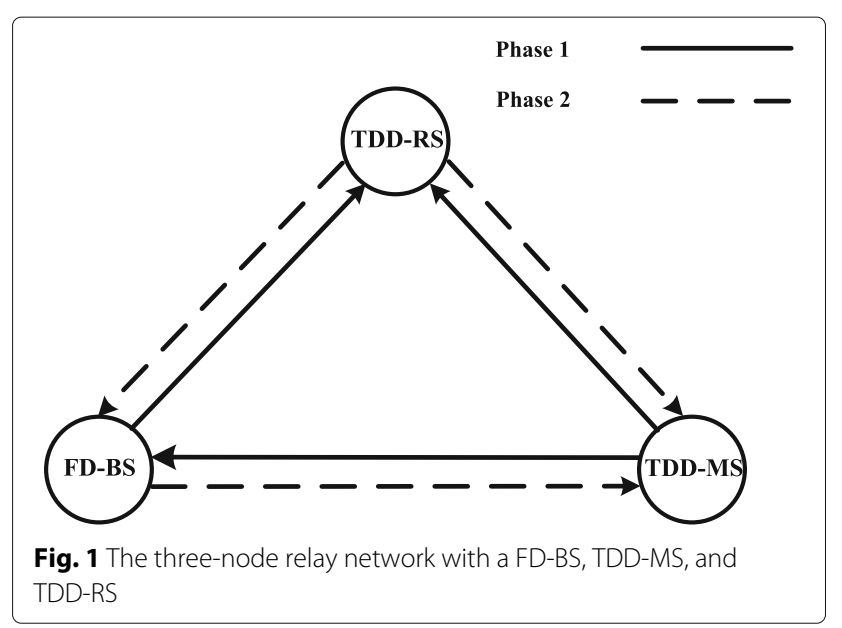


relaying schemes, we first model the signals at the first phase as follows.

It is assumed that the BS receiver cancels the interference from its own transmitter by jointly using three-step interference cancelation, i.e., antenna, analog, and digital interference cancelation. Recent experimental results show that the non-linear effect of the transmitter's amplifier is the bottleneck in interference cancelation, and thus the residual interference is mainly composed of non-linear component [2,13], which can be modeled as a Gaussian noise. Thus, the BS's received signal at the first phase can be expressed as

$$
y_{\mathrm{B}}[1]=\sqrt{P_{\mathrm{M}}} h_{\mathrm{BM}} x_{\mathrm{M}}[1]+n_{\mathrm{B}}[1],
$$

and the RS's received signal can be expressed as

$$
y_{\mathrm{R}}[1]=\sqrt{P_{\mathrm{M}}} h_{\mathrm{MR}} x_{\mathrm{M}}[1]+\sqrt{P_{\mathrm{B}}} h_{\mathrm{BR}} x_{\mathrm{B}}[1]+n_{\mathrm{R}}[1],
$$

where $x_{\mathrm{M}}[k]$ and $x_{\mathrm{B}}[k]$ denote the transmitted signals at the MS and BS, respectively, and $y_{\mathrm{B}}[k]$ and $y_{\mathrm{R}}[k]$ denote the received signals at the BS and RS at the $k$ th $(k=$ $1,2)$ phase, respectively. $h_{\mathrm{BM}}$ denotes the channel coefficient of direct link between the BS and MS, $h_{\mathrm{MR}}$ denotes the channel coefficient between the MS and RS, and $h_{\mathrm{BR}}$ denotes the channel coefficient between the BS and RS. $n_{\mathrm{B}}[k]$ denotes the residual SI plus thermal noise and $n_{\mathrm{R}}[k]$ denotes the additive white Gaussian noise (AWGN) at the RS's receiver, with $n_{\mathrm{B}}[k] \sim \mathcal{C N}\left(0, \sigma_{\mathrm{B}}^{2}\right), n_{\mathrm{R}}[k] \sim$ $\mathcal{C N}\left(0, \sigma_{\mathrm{R}}^{2}\right) . P_{\mathrm{M}}$ and $P_{\mathrm{B}}$ denote the average transmission power of the MS and BS, respectively.

In this paper, sum-capacity is used as the objective function, which is defined as the maximum summation of the achievable data rates of the uplink and downlink, and denoted by

$$
C=\max \left\{R_{\mathrm{UL}}+R_{\mathrm{DL}}\right\},
$$

where $C$ denotes the sum-capacity of the system. $R_{\mathrm{UL}}$ and $R_{\mathrm{DL}}$ denote the rate of uplink and downlink in the achievable rate region, respectively.

\section{The proposed AF relaying scheme}

In this section, we propose an AF relaying scheme with two phases for information exchange between the FD-BS and TDD-MS. The scheme is presented as follows.

At the first phase, the BS and MS transmit their signals to the RS. As the BS operates in FD mode, it can also receive the uplink signal from MS simultaneously. At the second phase, the RS amplifies and forwards the received signals to the BS and MS, and meanwhile, the BS transmits another downlink data stream to the MS. Thus, the MS receives two data streams, one from the RS and the other from the BS.

The proposed AF relaying scheme is similar to the traditional HD two-way relaying scheme [36], but with a few differences. For uplink, in the traditional AF relaying scheme, the BS only receive signals at the second phase. Whereas in the proposed scheme, as the BS works in FD mode, it can receive signals at both the first and the second phases, thus obtaining a receiver diversity gain. For downlink, in the traditional AF relaying scheme, the BS only transmit its signal at the first phase. However, in the proposed AF relaying scheme, the BS transmits downlink signal at two phases, thus obtaining a multiple transmission gain.

The received signals at the first phase can be expressed as (1) and (2) for BS and RS, respectively. At the second phase, the RS amplifies and forwards the received signal with transmission power $P_{\mathrm{R}}$. With the assumption that the channel coefficients are perfectly estimated, both the BS and MS can subtract their own transmission signals prior to decoding [37]. Therefore, at the second phase, the received signal at BS is

$$
y_{\mathrm{B}}[2]=\sqrt{P_{\mathrm{M}}} h_{\mathrm{BR}} h_{\mathrm{RM}} \rho x_{\mathrm{M}}[1]+h_{\mathrm{BR}} \rho n_{\mathrm{R}}[1]+n_{\mathrm{B}}[2],
$$

where $\rho$ is the amplify factor with

$$
\rho=\frac{\sqrt{P_{\mathrm{R}}}}{\sqrt{P_{\mathrm{M}}\left|h_{\mathrm{RM}}\right|^{2}+P_{\mathrm{B}}\left|h_{\mathrm{BR}}\right|^{2}+\sigma_{\mathrm{R}}^{2}}} .
$$

For uplink, as BS receives two copies of the signal transmitted from MS at two phases, the BS's receiver combines the received signals by using the criterion of maximum ratio combination (MRC) [38]. Thus, the uplink rate upper bound can be calculated by

$$
R_{\mathrm{UL}}^{\mathrm{AF}}<\gamma\left[\frac{P_{\mathrm{M}}\left|h_{\mathrm{BM}}\right|^{2}}{\sigma_{\mathrm{B}}^{2}}+\frac{P_{\mathrm{M}}\left|h_{\mathrm{RM}} h_{\mathrm{BR}} \rho\right|^{2}}{\sigma_{\mathrm{B}}^{2}+\sigma_{\mathrm{R}}^{2}\left|h_{\mathrm{BR}} \rho\right|^{2}}\right],
$$

where $\gamma[x]=\frac{1}{2} \log _{2}(1+x)$.

For downlink, the MS receives signals from both the BS and RS at the second phase, which is equivalent to a $2 \times$ 1 multiple-input single-output (MISO) system. Therefore, the downlink date rate is upper bounded by [39]

$$
R_{\mathrm{DL}}^{\mathrm{AF}}<\gamma\left[\frac{P_{\mathrm{B}}\left(\left|h_{\mathrm{BM}}\right|+\left|h_{\mathrm{RM}} h_{\mathrm{BR}} \rho\right|\right)^{2}}{\sigma_{\mathrm{M}}^{2}+\sigma_{\mathrm{R}}^{2}\left|h_{\mathrm{RM}} \rho\right|^{2}}\right],
$$

where $\sigma_{\mathrm{M}}^{2}$ denotes the noise power at the MSs receiver.

To achieve the maximum sum-rate, the BS and RS should jointly perform a beamforming to maximize the signal-to-noise ratio (SNR) at the MS's receiver [39], i.e., the downlink signal is transmitted from the BS and RS simultaneously, but with different phase rotations. With the assumption that the BS and RS perfectly know the CSI, they can adjust the phase rotations, so that the received signals from the BS and RS are in phase at the MSs receive 
antenna. Finally, according to (6) and (7), the sum-capacity of the proposed AF relaying scheme can be expressed as

$$
\begin{aligned}
C^{\mathrm{AF}}=\gamma & {\left[\frac{P_{\mathrm{M}}\left|h_{\mathrm{BM}}\right|^{2}}{\sigma_{\mathrm{B}}^{2}}+\frac{P_{\mathrm{M}}\left|h_{\mathrm{RM}} h_{\mathrm{BR}} \rho\right|^{2}}{\sigma_{\mathrm{B}}^{2}+\sigma_{\mathrm{R}}^{2}\left|h_{\mathrm{BR}} \rho\right|^{2}}\right] } \\
& +\gamma\left[\frac{P_{\mathrm{B}}\left(\left|h_{\mathrm{BM}}\right|+\left|h_{\mathrm{RM}} h_{\mathrm{BR}} \rho\right|\right)^{2}}{\sigma_{\mathrm{M}}^{2}+\sigma_{\mathrm{R}}^{2}\left|h_{\mathrm{RM}} \rho\right|^{2}}\right] .
\end{aligned}
$$

\section{The proposed DF relaying scheme}

\subsection{Multiple DF relaying modes selection criterion for sum-capacity maximization}

In designing the DF relaying scheme, a key issue is to design the decode-and-forward mode at the second phase for the RS. During the second phase, the RS can decode and forward the data streams from both the BS and MS, only from BS, only from MS, or none of them, which are referred to as bidirectional DF (BDF) mode, downlink DF (DDF) mode, uplink DF (UDF) mode, and no DF (NF) mode, respectively.

Let us denote the SNRs of the channels from the BS to RS, from the BS to MS, and from the MR to RS by

$$
\begin{aligned}
\mathrm{SNR}_{\mathrm{BR}} & =\frac{P_{\mathrm{B}} \cdot\left|h_{\mathrm{BR}}\right|^{2}}{\sigma_{\mathrm{R}}^{2}}, \\
\mathrm{SNR}_{\mathrm{BM}} & =\frac{P_{\mathrm{B}} \cdot\left|h_{\mathrm{BM}}\right|^{2}}{\sigma_{\mathrm{M}}^{2}}, \\
\mathrm{SNR}_{\mathrm{MR}} & =\frac{P_{\mathrm{M}} \cdot\left|h_{\mathrm{BM}}\right|^{2}}{\sigma_{\mathrm{R}}^{2}},
\end{aligned}
$$

respectively.

For the case that $\mathrm{SNR}_{\mathrm{BM}}$ is much smaller than $\mathrm{SNR}_{\mathrm{MR}}$ and $\mathrm{SNR}_{\mathrm{BR}}$, the RS can dramatically improve the performances of both the uplink and downlink; therefore, the $\mathrm{BDF}$ is the best among the four modes. Contrarily, for the case that $\mathrm{SNR}_{M R}$ and $\mathrm{SNR}_{\mathrm{BR}}$ are much smaller than $\mathrm{SNR}_{\mathrm{BM}}$, the RS is unnecessary, thus direct transmission between the BS and MS, i.e., the NF mode, is the best. The DDF mode has its advantage if $\mathrm{SNR}_{M R}$ is much smaller than $S_{N R}$ BM and $S N R_{B R}$. In this case, the uplink data is only transmitted directly from MS to BS at the first phase, without the assistance of RS. Consequently, the uplink data rate is not limited by the channel fading from MS to RS, which would be a limiting factor to the capacity when $\mathrm{SNR}_{\mathrm{MR}}$ is very small. Similarly, the UDF mode offers the maximum capacity if $S N R_{B R}$ is much smaller than $S N R_{B M}$ and $S_{N R}$ MR. In this case, as $S_{N R} R_{B R}$ is very small, to decode the downlink data at RS requires a very low coding rate, thus limiting the uplink capacity. Therefore, the best strategy is to decode and forward only the uplink data at RS, i.e., the UDF mode.

Selecting the best relaying mode from the above four modes, the system can obtain a selection diversity gain. In this paper, we propose a multiple relaying modes selection criterion to maximize the sum-capacity. The proposed criterion can be expressed as

$$
\Re^{*}=\underset{\Re \in \Phi}{\arg \max } C_{\Re},
$$

where $\Phi=\{\mathrm{BDF}, \mathrm{DDF}, \mathrm{UDF}, \mathrm{NF}\}$ denotes the relaying mode set and $C_{\Re}$ denotes the sum-capacity of mode $\Re$. In the following subsections, we will present the achievable rate region, the sum-capacity, and the transmission and reception modes to achieve the sum-capacity for the four relaying modes.

\subsection{Bidirectional DF mode}

In the BDF mode, the RS decodes both the uplink and downlink data from the received signal by using a successive decoding method, in which the RS first decodes one data stream while treating the other one as a noise, and then subtracts the decoded signal from the received signal, thus then decodes the rest data stream without any interference. After that, the RS encodes the data stream using network coding, i.e., performs an XOR operation on the decoded information bits from BS and MS. Finally, the RS broadcasts them to BS and MS. Meanwhile, the BS transmits another independent downlink signal, which is shown in Fig. 2.

At the first phase, the relay channel can be modeled as a multiple access channel (MAC) [40], therefore, the capacity region can be given by

$$
\left\{\begin{array}{l}
R_{\mathrm{UL}}^{\mathrm{BDF}}[1]<\gamma\left[\frac{P_{\mathrm{M}}\left|h_{\mathrm{MR}}\right|^{2}}{\sigma_{\mathrm{R}}^{2}}\right], \\
R_{\mathrm{DL}}^{\mathrm{BDF}}[1]<\gamma\left[\frac{P_{\mathrm{B}}\left|h_{\mathrm{BR}}\right|^{2}}{\sigma_{\mathrm{R}}^{2}}\right], \\
R_{\mathrm{DL}}^{\mathrm{BDF}}[1]+R_{\mathrm{UL}}^{\mathrm{BDF}}[1]<\gamma\left[\frac{P_{\mathrm{M}}\left|h_{\mathrm{MR}}\right|^{2}+P_{\mathrm{B}}\left|h_{\mathrm{BR}}\right|^{2}}{\sigma_{\mathrm{R}}^{2}}\right] .
\end{array}\right.
$$

The above upper bound can be achieved by using superposition coding with time sharing or rate splitting at transmitter and the successive decoding at receiver [41]. For uplink, the BS decodes the uplink data by jointly using the received signals at the first and second phases (Fig. 3). Therefore, the achievable uplink rate is upper bounded by [42]

$$
R_{\mathrm{UL}}^{\mathrm{BDF}}[2]<\gamma\left[\frac{P_{\mathrm{M}}\left|h_{\mathrm{BM}}\right|^{2}}{\sigma_{\mathrm{B}}^{2}}\right]+\gamma\left[\frac{P_{\mathrm{R}}\left|h_{\mathrm{BR}}\right|^{2}}{\sigma_{\mathrm{B}}^{2}}\right] .
$$

For downlink, as MS receives two signals from the BS and the RS, respectively. At the second phase, the capacity region can be expressed as

$$
\left\{\begin{array}{l}
R_{\mathrm{BS}-\mathrm{MS}}^{\mathrm{BDF}}[2]<\gamma\left[\frac{P_{\mathrm{B}}\left|h_{\mathrm{BM}}\right|^{2}}{\sigma_{\mathrm{M}}^{2}}\right] \\
R_{\mathrm{RS}-\mathrm{MS}}^{\mathrm{BDF}}[2]<\gamma\left[\frac{P_{\mathrm{R}}\left|h_{\mathrm{MR}}\right|^{2}}{\sigma_{\mathrm{M}}^{2}}\right] \\
R_{\mathrm{BS}-\mathrm{MS}}^{\mathrm{BDF}}[2]+R_{\mathrm{RS}-\mathrm{MS}}^{\mathrm{BDF}}[2]<\gamma\left[\frac{P_{\mathrm{B}}\left|h_{\mathrm{BM}}\right|^{2}+P_{\mathrm{R}}\left|h_{\mathrm{MR}}\right|^{2}}{\sigma_{\mathrm{M}}^{2}}\right]
\end{array}\right.
$$



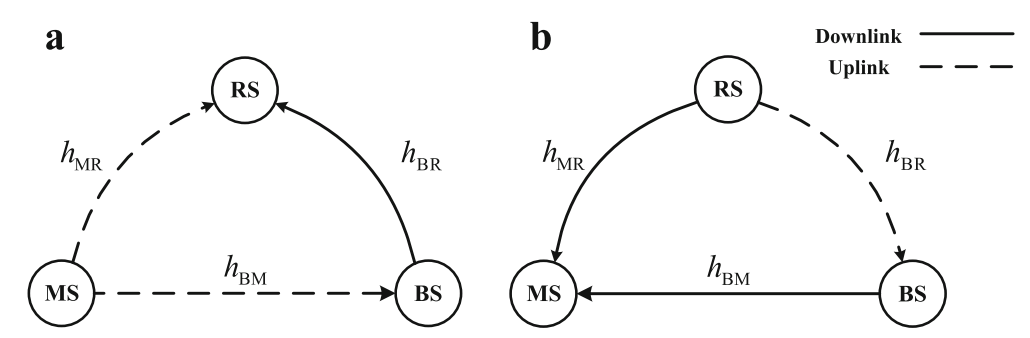

Fig. 2 Bidirectional DF relaying scheme. a the first phase, $\mathbf{b}$ the second phase

where $R_{\mathrm{RS}-\mathrm{MS}}^{\mathrm{BDF}}[2]$ denotes the rate from the RS to MS and $R_{\mathrm{BS}-\mathrm{MS}}^{\mathrm{BDF}}[2]$ denotes the rate from the $\mathrm{BS}$ to $\mathrm{MS}$ at the second phase. Due to that, the forward information bits from the RS to MS at the second phase should be less than or equal to the transmitted bits from the BS at the first phase, and $R_{\mathrm{BS}-\mathrm{MS}}^{\mathrm{BDF}}[2]+R_{\mathrm{RS}-\mathrm{MS}}^{\mathrm{BDF}}[2]=R_{\mathrm{DL}}^{\mathrm{BDF}}[2]$, the downlink data rate at the second phase is upper bounded by

$$
\left\{\begin{array}{l}
R_{\mathrm{DL}}^{\mathrm{BDF}}[2]<R_{\mathrm{DL}}^{\mathrm{BDF}}[1]+\gamma\left[\frac{P_{\mathrm{B}}\left|h_{\mathrm{BM}}\right|^{2}}{\sigma_{\mathrm{M}}^{2}}\right] \\
\text { if } R_{\mathrm{DL}}^{\mathrm{BDF}}[1]<\gamma\left[\frac{P_{\mathrm{R}}\left|h_{\mathrm{MR}}\right|^{2}}{\sigma_{\mathrm{M}}^{2}+P_{\mathrm{B}}\left|h_{\mathrm{BM}}\right|^{2}}\right] \\
R_{\mathrm{DL}}^{\mathrm{BDF}}[2]<\gamma\left[\frac{P_{\mathrm{B}}\left|h_{\mathrm{BM}}\right|^{2}+P_{\mathrm{R}}\left|h_{\mathrm{MR}}\right|^{2}}{\sigma_{\mathrm{M}}^{2}}\right], \quad \text { otherwise. }
\end{array}\right.
$$

Taking consideration of all the upper bounds in (11), (12), and (14), rate regions in terms of $R_{\mathrm{DL}}, R_{\mathrm{UL}}$, and $R_{\mathrm{UL}}+R_{\mathrm{DL}}$ can be obtained by using the Fourier-Motzkin elimination (FME) method (see Appendix D of [43]). The achievable rate region is finally given by

$$
\left\{\begin{array}{l}
R_{\mathrm{UL}}^{\mathrm{BDF}}<\min \left\{\gamma\left[\frac{P_{\mathrm{M}}\left|h_{\mathrm{MR}}\right|^{2}}{\sigma_{\mathrm{R}}^{2}}\right], \gamma\left[\frac{P_{\mathrm{M}}\left|h_{\mathrm{BM}}\right|^{2}}{\sigma_{\mathrm{B}}^{2}}\right]+\gamma\left[\frac{P_{\mathrm{R}}\left|h_{\mathrm{BR}}\right|^{2}}{\sigma_{\mathrm{B}}^{2}}\right]\right\}, \\
R_{\mathrm{DL}}^{\mathrm{BDF}}<\gamma\left[\frac{P_{\mathrm{B}}\left|h_{\mathrm{BM}}\right|^{2}}{\sigma_{\mathrm{M}}^{2}}\right]+\min \left\{\gamma\left[\frac{P_{\mathrm{B}}\left|h_{\mathrm{BR}}\right|^{2}}{\sigma_{\mathrm{R}}^{2}}\right], \gamma\left[\frac{P_{\mathrm{R}}\left|h_{\mathrm{MR}}\right|^{2}}{\sigma_{\mathrm{M}}^{2}+P_{\mathrm{B}}\left|h_{\mathrm{BM}}\right|^{2}}\right]\right\}, \\
R_{\mathrm{DL}}^{\mathrm{BDF}}+R_{\mathrm{UL}}^{\mathrm{BDF}}<\gamma\left[\frac{P_{\mathrm{M}}\left|h_{\mathrm{MR}}\right|^{2}+P_{\mathrm{B}}\left|h_{\mathrm{BR}}\right|^{2}}{\sigma_{\mathrm{R}}^{2}}\right] .
\end{array}\right.
$$

It is observed from (15) that the discussion of the achievable rate region depends on two special boundaries, which are represented by

$$
\begin{aligned}
& \beta_{1}=\gamma\left[\frac{P_{\mathrm{R}}\left|h_{\mathrm{MR}}\right|^{2}}{\sigma_{\mathrm{M}}^{2}+P_{\mathrm{B}}\left|h_{\mathrm{BM}}\right|^{2}}\right], \\
& \beta_{2}=\gamma\left[\frac{P_{\mathrm{M}}\left|h_{\mathrm{BM}}\right|^{2}}{\sigma_{\mathrm{B}}^{2}}\right]+\gamma\left[\frac{P_{\mathrm{R}}\left|h_{\mathrm{BR}}\right|^{2}}{\sigma_{\mathrm{B}}^{2}}\right] .
\end{aligned}
$$

According to different channel conditions, the rate region is bounded in four cases as shown in Fig. 4, and the sum-capacity can be achieved at the boundary points, $C_{i} \quad(i=1,2,3,4)$. Finally, the sum-capacity of the BDF mode can be written by

$$
C^{\mathrm{BDF}}=\min \left\{C_{\mathrm{UL}}^{\mathrm{BDF}}+C_{\mathrm{DL}}^{\mathrm{BDF}}, \gamma\left[\frac{P_{\mathrm{M}}\left|h_{\mathrm{MR}}\right|^{2}+P_{\mathrm{B}}\left|h_{\mathrm{BR}}\right|^{2}}{\sigma_{\mathrm{R}}^{2}}\right]\right\},
$$

where

$$
\begin{aligned}
& C_{\mathrm{UL}}^{\mathrm{BDF}}= \min \left\{\gamma\left[\frac{P_{\mathrm{M}}\left|h_{\mathrm{MR}}\right|^{2}}{\sigma_{\mathrm{R}}^{2}}\right], \gamma\left[\frac{P_{\mathrm{M}}\left|h_{\mathrm{BM}}\right|^{2}}{\sigma_{\mathrm{B}}^{2}}\right]+\gamma\left[\frac{P_{\mathrm{R}}\left|h_{\mathrm{BR}}\right|^{2}}{\sigma_{\mathrm{B}}^{2}}\right]\right\}, \\
& C_{\mathrm{DL}}^{\mathrm{BDF}}=\min \left\{\gamma\left[\frac{P_{\mathrm{B}}\left|h_{\mathrm{BR}}\right|^{2}}{\sigma_{\mathrm{R}}^{2}}\right]+\gamma\left[\frac{P_{\mathrm{B}}\left|h_{\mathrm{BM}}\right|^{2}}{\sigma_{\mathrm{M}}^{2}}\right],\right. \\
&\left.\gamma\left[\frac{P_{\mathrm{R}}\left|h_{\mathrm{MR}}\right|^{2}+P_{\mathrm{B}}\left|h_{\mathrm{BM}}\right|^{2}}{\sigma_{\mathrm{M}}^{2}}\right]\right\} .
\end{aligned}
$$
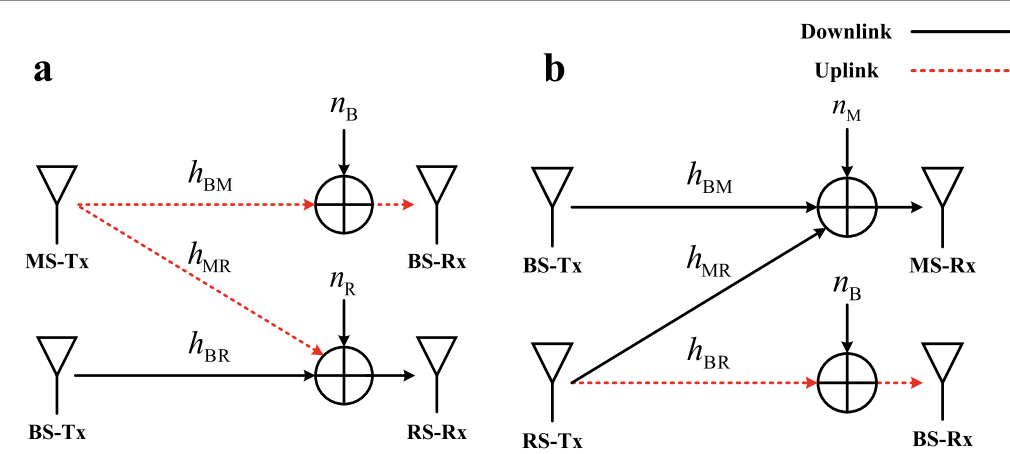

Fig. 3 The system model of a two-hop transmission link: $\mathbf{a}$ the first phase, and $\mathbf{b}$ the second phase 

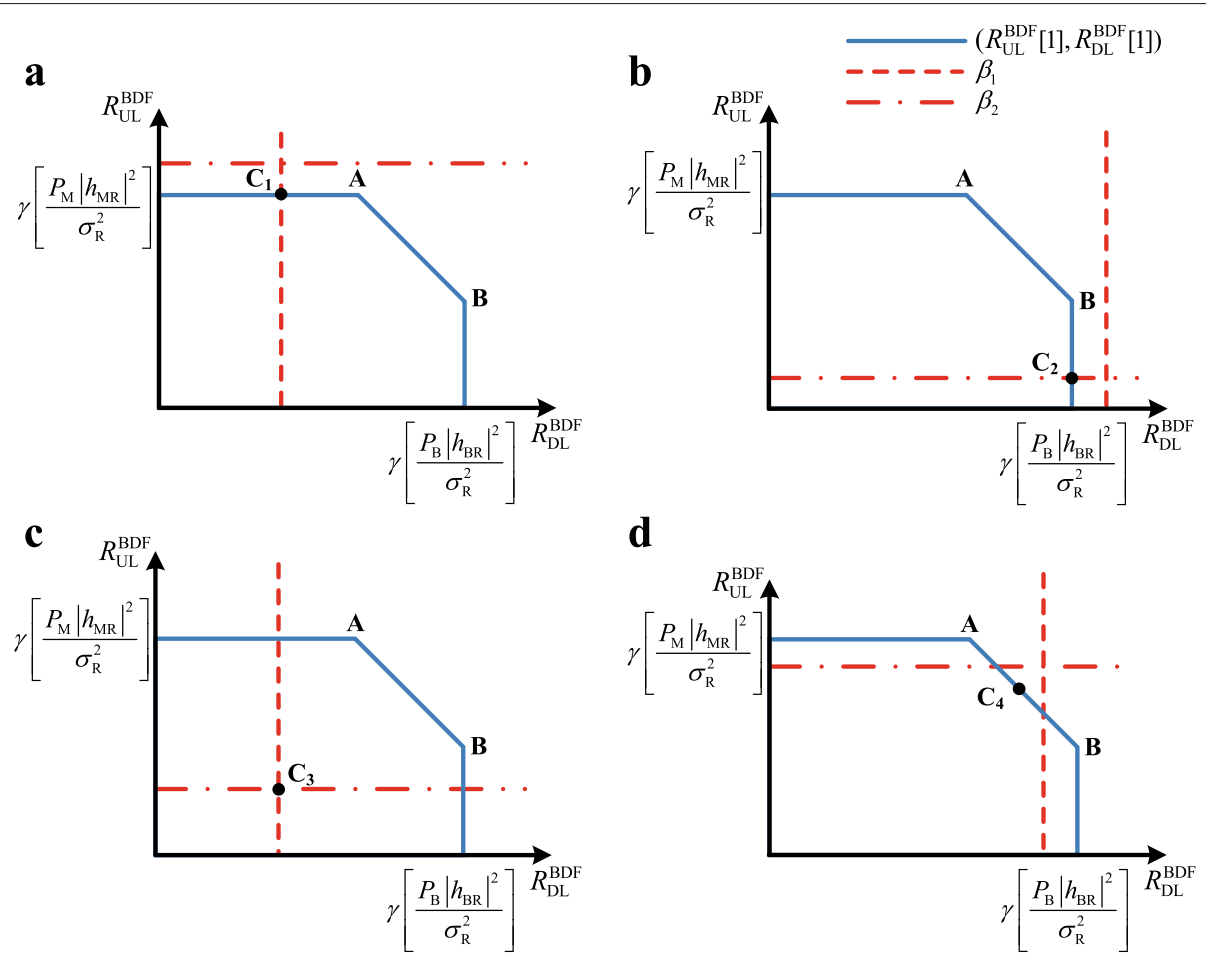

d

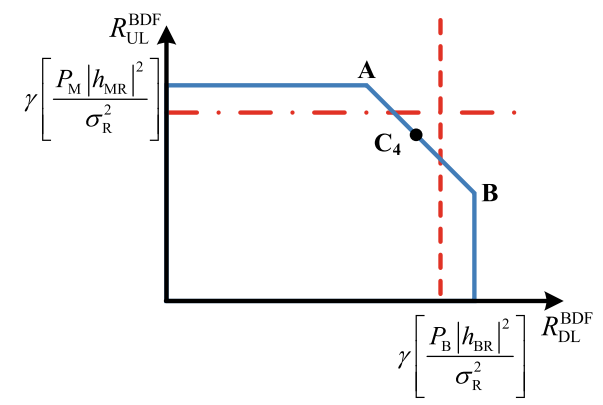

Fig. 4 The achievable rate regions and sum-capacities of the BDF relaying mode for four conditions. a $\beta_{1}<\gamma\left[P_{B}\left|h_{B R}\right|^{2} /\left(\sigma_{R}^{2}+P_{M}\left|h_{M R}\right|^{2}\right)\right]$, $\beta_{2}>\gamma\left(P_{\mathrm{M}}\left|h_{\mathrm{MR}}\right|^{2} / \sigma_{\mathrm{R}}^{2}\right) \cdot \mathbf{b} \beta_{1}>\gamma\left(P_{\mathrm{B}}\left|h_{\mathrm{BR}}\right|^{2} / \sigma_{\mathrm{R}}^{2}\right), \beta_{2}<\gamma\left[P_{\mathrm{M}}\left|h_{\mathrm{MR}}\right|^{2} /\left(\sigma_{\mathrm{R}}^{2}+P_{\mathrm{B}}\left|h_{\mathrm{BR}}\right|^{2}\right)\right] . \mathbf{c} \beta_{1}<\gamma\left[P_{\mathrm{B}}\left|h_{\mathrm{BR}}\right|^{2} /\left(\sigma_{\mathrm{R}}^{2}+P_{\mathrm{M}}\left|h_{\mathrm{MR}}\right|^{2}\right)\right]$, $\beta_{2}<\gamma\left[P_{M}\left|h_{M R}\right|^{2} /\left(\sigma_{R}^{2}+P_{B}\left|h_{B R}\right|^{2}\right)\right] \cdot d \beta_{1}+\beta_{2}>\gamma\left[\left(P_{M}\left|h_{M R}\right|^{2}+P_{B}\left|h_{B R}\right|^{2}\right) / \sigma_{R}^{2}\right]$

For cases a, b, and c in Fig. 4, the sum-capacity is achieved only at one point, i.e., there is only one optimal uplink and downlink rate pair; however, for case $\mathrm{d}$, the sum-capacity is achieved for any rate pair on the segment of

$$
R_{\mathrm{DL}}^{\mathrm{BDF}}+R_{\mathrm{UL}}^{\mathrm{BDF}}<\gamma\left[\frac{P_{\mathrm{M}}\left|h_{\mathrm{MR}}\right|^{2}+P_{\mathrm{B}}\left|h_{\mathrm{BR}}\right|^{2}}{\sigma_{\mathrm{R}}^{2}}\right] .
$$

\subsection{Downlink DF mode}

In the DDF Mode, the RS decodes and forwards only the downlink signal. At the first phase, as shown in Fig. 3, the link from MS to RS is an interference link. This can be modeled as the so-called Z-channel [44], in which the $B S$ received signal is interference-free, whereas the RS receives a combination of the desired downlink signal and the interfering signal. The received signals at the first phase in (1) and (2) can be rewritten in a standard form of Gaussian Z-channel [45] as

$$
\begin{array}{r}
y_{\mathrm{B}}^{*}=\sqrt{\frac{P_{\mathrm{M}}}{\sigma_{\mathrm{B}}^{2}}} h_{\mathrm{BM}} x_{\mathrm{M}}[1]+n_{\mathrm{B}}^{*}, \\
y_{\mathrm{R}}^{*}=\sqrt{\frac{P_{\mathrm{B}}}{\sigma_{\mathrm{R}}^{2}}} h_{\mathrm{BR}} x_{\mathrm{B}}[1]+\sqrt{\frac{P_{\mathrm{M}}}{\sigma_{\mathrm{B}}^{2}}} h_{\mathrm{BM}} \eta_{\mathrm{inf} x_{\mathrm{M}}[1]+n_{\mathrm{R}}^{*},}
\end{array}
$$

where $n_{\mathrm{B}}^{*}$ and $n_{\mathrm{R}}^{*}$ are additive Gaussian noises with zero mean and unit variance and $\eta_{\text {inf }}$ denotes the interference coefficient defined as $\eta_{\text {inf }}=\frac{h_{\mathrm{MR}} \cdot \sigma_{\mathrm{B}}}{h_{\mathrm{BM}} \cdot \sigma_{\mathrm{R}}}$.

The rate region of the Gaussian $Z$-channel is upper bounded by [46]. We have

$$
\left\{\begin{array}{rl}
R_{\mathrm{UL}}^{\mathrm{DDF}}[1]<\gamma\left[\frac{P_{\mathrm{M}}\left|h_{\mathrm{BM}}\right|^{2}}{\sigma_{\mathrm{B}}^{2}}\right] & ; \\
R_{\mathrm{DL}}^{\mathrm{DDF}}[1]<\gamma\left[\frac{P_{\mathrm{B}}\left|h_{\mathrm{BR}}\right|^{2}}{\sigma_{\mathrm{R}}^{2}}\right] ; & R_{\mathrm{UL}}^{\mathrm{DDF}}[1]+R_{\mathrm{DL}}^{\mathrm{DDF}}[1]<\gamma\left[\frac{P_{\mathrm{B}}\left|h_{\mathrm{BR}}\right|^{2}+P_{\mathrm{M}}\left|h_{\mathrm{MR}}\right|^{2}}{\sigma_{\mathrm{R}}^{2}}\right] \\
& +\frac{1}{2} \log _{2}\left(\frac{P_{\mathrm{M}}\left|h_{\mathrm{BM}}\right|^{2}+\sigma_{\mathrm{B}}^{2}}{\min \left\{\eta_{\mathrm{inf}}, 1\right\} \cdot P_{\mathrm{M}}\left|h_{\mathrm{BM}}\right|^{2}+\sigma_{\mathrm{B}}^{2}}\right)
\end{array} .\right.
$$

At the second phase, the SNR of the received signal at the MS is maximized by applying beamforming technique, thus the downlink rate is upper bounded by

$$
R_{\mathrm{DL}}^{\mathrm{DDF}}[2]<\gamma\left[\frac{\left(\sqrt{P_{\mathrm{B}}} h_{\mathrm{BM}}+\sqrt{P_{\mathrm{R}}} h_{\mathrm{MR}}\right)^{2}}{\sigma_{\mathrm{M}}^{2}}\right] .
$$



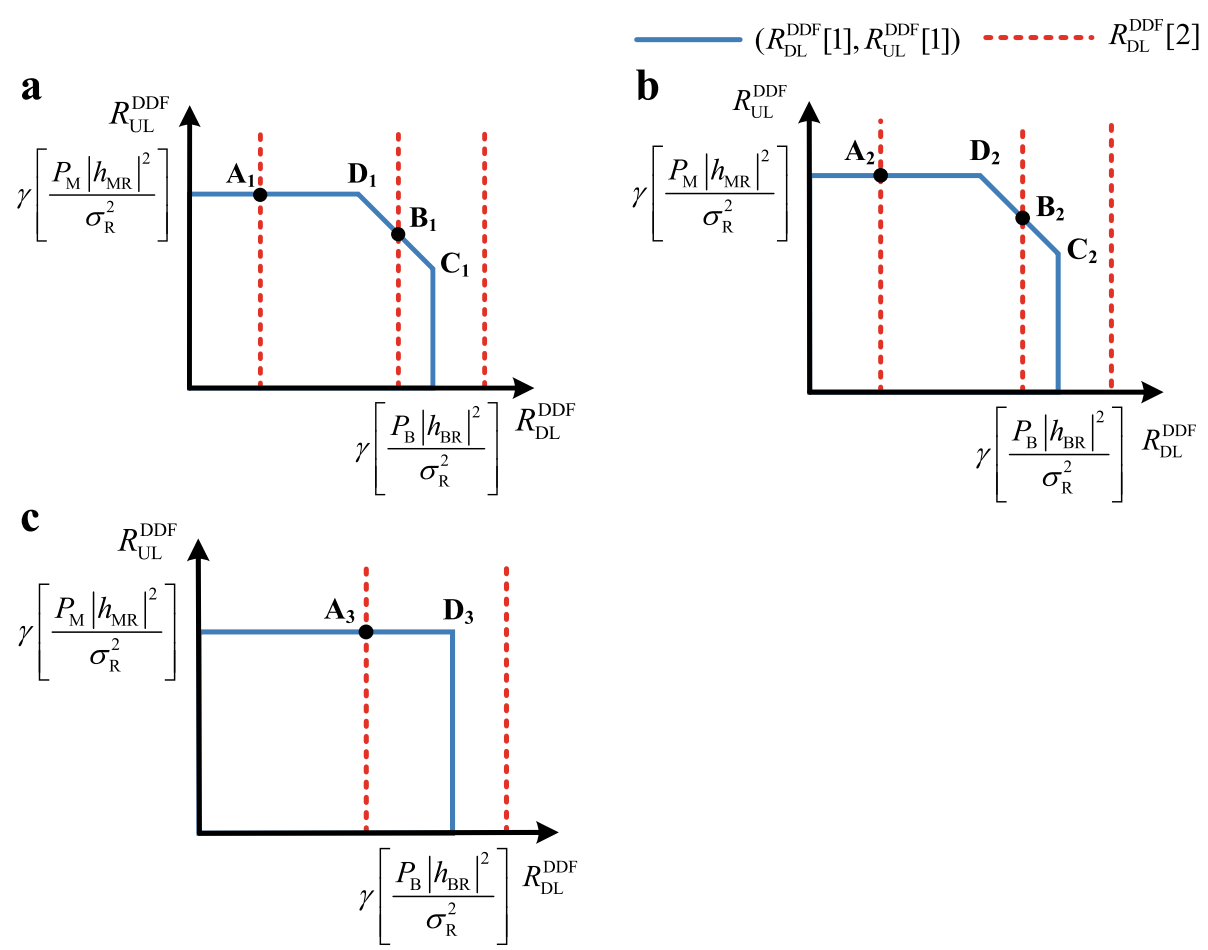

Fig. 5 The achievable rate regions and sum-capacities of DDF relaying mode for different interferences cases. a Weak or moderate interference $\left(0 \leq \eta_{\text {inf }} \leq 1\right)$. b Strong interference $\left(1 \leq \eta_{\text {inf }} \leq \sqrt{1+P_{B}\left|h_{B R}\right|^{2} / \sigma_{R}^{2}}\right)$. c Very strong interference $\left(\eta_{\text {inf }} \geq \sqrt{1+P_{B}\left|h_{B R}\right|^{2} / \sigma_{R}^{2}}\right)$

According to (22) and (23), the achievable rate region is

$$
\left\{\begin{array}{l}
R_{\mathrm{UL}}^{\mathrm{DDF}}<\gamma\left[\frac{P_{\mathrm{M}}\left|h_{\mathrm{BM}}\right|^{2}}{\sigma_{\mathrm{B}}^{2}}\right] \\
R_{\mathrm{DL}}^{\mathrm{DDF}}<\min \left\{\gamma\left[\frac{P_{\mathrm{B}}\left|h_{\mathrm{BR}}\right|^{2}}{\sigma_{\mathrm{R}}^{2}}\right], \gamma\left[\frac{\left(\sqrt{P_{\mathrm{B}}} h_{\mathrm{BM}}+\sqrt{P_{\mathrm{R}}} h_{\mathrm{MR}}\right)^{2}}{\sigma_{\mathrm{M}}^{2}}\right]\right\} \\
R_{\mathrm{UL}}^{\mathrm{DDF}}+R_{\mathrm{DL}}^{\mathrm{DDF}}=R_{\mathrm{UL}}^{\mathrm{DDF}}[1]+R_{\mathrm{DL}}^{\mathrm{DDF}}[1] .
\end{array}\right.
$$

The sum-capacity of the first phase can be expressed in three cases according to the value of $\eta_{\text {inf. Figure } 5 \text { shows }}$ the relationship between the upper bound of $Z$-channel in (22) and the upper bound of (23) for the three cases of $Z$-channel. By taking the minimum upper bound for each case, the sum-capacity $C^{\mathrm{DDF}}$ can be expressed as where $R_{\mathrm{DL}}^{\mathrm{DDF}}[2]$ is given by (23).

\subsection{Uplink DF mode}

In the UDF relaying mode, at the second phase, the RS only decodes the uplink signal, and then re-encodes and forwards it. Meanwhile, the BS transmits another downlink signal.

At the first phase, the RS detects the uplink signal by treating the downlink signal as an additive Gaussian noise; therefore, the uplink data rate should be upper bounded by

$$
R_{\mathrm{UL}}^{\mathrm{UDF}}[1]<\gamma\left[\frac{P_{\mathrm{M}}\left|h_{\mathrm{MR}}\right|^{2}}{\sigma_{\mathrm{B}}^{2}+P_{\mathrm{B}}\left|h_{\mathrm{BR}}\right|^{2}}\right] .
$$

Note that the assumption of Gaussian distribution used here is only for capacity analysis, but not a mandatory

$$
C^{\mathrm{DDF}}= \begin{cases}\gamma\left(\frac{P_{\mathrm{M}}\left|h_{\mathrm{BM}}\right|^{2}}{\sigma_{\mathrm{B}}^{2}}\right)+\min \left\{\gamma\left(\frac{P_{\mathrm{B}}\left|h_{\mathrm{BR}}\right|^{2}}{\sigma_{\mathrm{R}}^{2}+P_{\mathrm{M}}\left|h_{\mathrm{MR}}\right|^{2}}\right), R_{\mathrm{DL}}^{\mathrm{DDF}}[2]\right\}, & \text { if } \eta_{\text {inf }} \leq 1 ; \\ \gamma\left(\frac{P_{\mathrm{M}}\left|h_{\mathrm{MR}}\right|^{2}}{\sigma_{\mathrm{R}}^{2}}\right)+\min \left\{\gamma\left(\frac{P_{\mathrm{B}}\left|h_{\mathrm{BR}}\right|^{2}}{\sigma_{\mathrm{R}}^{2}+P_{\mathrm{M}}\left|h_{\mathrm{MR}}\right|^{2}}\right), R_{\mathrm{DL}}^{\mathrm{DDF}}[2]\right\}, & \text { if } 1 \leq \eta_{\mathrm{inf}} \leq \sqrt{1+\frac{P_{\mathrm{B}}\left|h_{\mathrm{BR}}\right|^{2}}{\sigma_{\mathrm{R}}^{2}}} \\ \gamma\left(\frac{P_{\mathrm{M}}\left|h_{\mathrm{BM}}\right|^{2}}{\sigma_{\mathrm{B}}^{2}}\right)+\min \left\{\gamma\left(\frac{P_{\mathrm{B}}\left|h_{\mathrm{BR}}\right|^{2}}{\sigma_{\mathrm{R}}^{2}}\right), R_{\mathrm{DL}}^{\mathrm{DDF}}[2]\right\}, & \text { if } \eta_{\text {inf }} \geq \sqrt{1+\frac{P_{\mathrm{B}}\left|h_{\mathrm{BR}}\right|^{2}}{\sigma_{\mathrm{R}}^{2}}}\end{cases}
$$


requirement for practical application of the proposed scheme. At the second phase, the RS forwards the decoded uplink signal, and the MS receives the downlink signal from the BS. As the MS knows the forwarded uplink signal from RS, the MS subtracts it from the received signal, thus the MS's received signal is interference-free. Therefore, the channel in Fig. 3b can be transformed to a parallel fading channel.

The BS recovers the uplink message by using the signals received at the first and second phases. The downlink signal is only transmitted and received at the second phase without the assistance of RS. Thus, the uplink and downlink rates are expressed as

$$
\left\{\begin{array}{l}
R_{\mathrm{UL}}^{\mathrm{UDF}}[2]<\gamma\left[\frac{P_{\mathrm{M}}\left|h_{\mathrm{BM}}\right|^{2}}{\sigma_{\mathrm{B}}^{2}}\right]+\gamma\left[\frac{P_{\mathrm{R}}\left|h_{\mathrm{BR}}\right|^{2}}{\sigma_{\mathrm{R}}^{2}}\right] \\
R_{\mathrm{DL}}^{\mathrm{UDF}}[2]<\gamma\left[\frac{P_{\mathrm{B}}\left|h_{\mathrm{BM}}\right|^{2}}{\sigma_{\mathrm{M}}^{2}}\right]
\end{array} .\right.
$$

The achievable rate region of UDF mode is

$$
\left\{\begin{array}{l}
R_{\mathrm{DL}}^{\mathrm{UDF}}=R_{\mathrm{DL}}^{\mathrm{UDF}}[2] \\
R_{\mathrm{UL}}^{\mathrm{UDF}}=\min \left\{R_{\mathrm{UL}}^{\mathrm{UDF}}[1], R_{\mathrm{UL}}^{\mathrm{UDF}}[2]\right\}
\end{array} .\right.
$$

The sum-capacity is achieved when both the uplink and downlink data rates are maximized and can be expressed as

$$
\begin{aligned}
C^{\mathrm{UDF}}=\gamma & {\left[\frac{P_{\mathrm{B}}\left|h_{\mathrm{BM}}\right|^{2}}{\sigma_{\mathrm{M}}^{2}}\right]+\min \left\{\gamma\left[\frac{P_{\mathrm{M}}\left|h_{\mathrm{MR}}\right|^{2}}{\sigma_{\mathrm{B}}^{2}+P_{\mathrm{B}}\left|h_{\mathrm{BR}}\right|^{2}}\right],\right.} \\
& \left.\gamma\left[\frac{P_{\mathrm{M}}\left|h_{\mathrm{BM}}\right|^{2}}{\sigma_{\mathrm{B}}^{2}}\right]+\left[\frac{P_{\mathrm{R}}\left|h_{\mathrm{BR}}\right|^{2}}{\sigma_{\mathrm{M}}^{2}}\right]\right\} .
\end{aligned}
$$

The rate region for UDF mode is shown in Fig. 6, in which point $\mathrm{A}$ or $\mathrm{D}$ denotes the achievable point with the maximum sum-capacity.

\subsection{No DF mode}

In the NF mode, the BS and MS exchange their information directly without the assistance of RS. The uplink

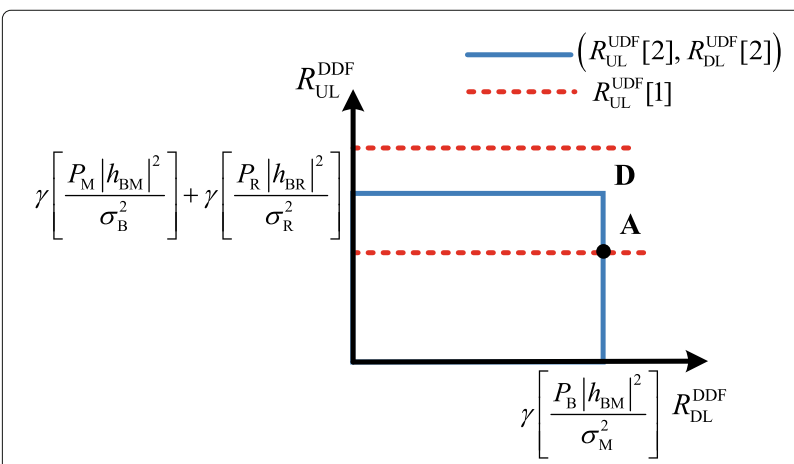

Fig. 6 The achievable rate regions and sum-capacities of UDF relaying mode signal is transmitted at the first phase, and the downlink signal is transmitted at the second phase. The sumcapacity is

$$
C^{\mathrm{NF}}=\gamma\left[\frac{P_{\mathrm{M}}\left|h_{\mathrm{BM}}\right|^{2}}{\sigma_{\mathrm{B}}^{2}}\right]+\gamma\left[\frac{P_{\mathrm{B}}\left|h_{\mathrm{BM}}\right|^{2}}{\sigma_{\mathrm{M}}^{2}}\right] .
$$

\section{Simulation results}

In this section, simulations are carried out to evaluate the performance of the proposed relaying schemes. To measure the performance advantages, we define capacity gain as

$$
G=\left(C-C^{\mathrm{NF}}\right) / C^{\mathrm{NF}},
$$

where $C$ is the capacity of the proposed AF or DF relaying schemes. We use the parameter $G$, whose unit is times, to evaluate the capacity improvement offered by RS. Accordingly, the capacity gains of $\mathrm{AF}$ and $\mathrm{DF}$ are denoted by $G_{\mathrm{AF}}$ and $G_{\mathrm{DF}}$, respectively.

Considering the reciprocal property of the channels and assuming equal signal power for all transmitters and equal noise power for all receivers, i.e., $P_{M}=P_{B}=P_{R}=P$ and $\sigma_{\mathrm{B}}^{2}=\sigma_{\mathrm{M}}^{2}=\sigma_{\mathrm{R}}^{2}=\sigma^{2}$. So we have $\mathrm{SNR}_{\mathrm{BR}}=\mathrm{SNR}_{\mathrm{RB}}$, $\mathrm{SNR}_{\mathrm{BM}}=\mathrm{SNR}_{\mathrm{MB}}$, and $\mathrm{SNR}_{\mathrm{MR}}=\mathrm{SNR}_{\mathrm{RM}}$. Thus, the channel conditions can be represented by these three factors, i.e., $\mathrm{SNR}_{\mathrm{BR}}$, $\mathrm{SNR}_{\mathrm{BM}}$, and $\mathrm{SNR}_{\mathrm{MR}}$. With (8), (17), (25), (26), (30), and (31), the sum-capacities of the AF relaying scheme and the four DF relaying modes can be seen as functions of $\mathrm{SNR}_{\mathrm{BR}}$, $\mathrm{SNR}_{\mathrm{BM}}$, and $\mathrm{SNR}_{\mathrm{MR}}$.

The capacity gain of the proposed AF relaying scheme is shown in Fig. 7. From Fig. 7, one can find that for lower SNR region, e.g., the lower left corner in Fig. 7a, the capacity gain is less than or equal to zero, which means that the AF relaying scheme is worse than the scheme without using RS when the SNRs of all the channels are very low. This is because in the AF relaying scheme, the RS amplifies the noise, thus leading to performance degradation. However, if one of the SNRs is larger enough, the capacity gain is positive, which means that the proposed AF relaying scheme has benefit compared with the NF mode. In addition, one can also find that the capacity gain obtained by using the proposed AF relaying scheme is more significant for a small value of $\mathrm{SNR}_{\mathrm{BM}}$ as shown in Fig. 7a, compared with that of a large value of $\mathrm{SNR}_{\mathrm{BM}}$, as shown in Fig. 7b. This is because that for smaller $\mathrm{SNR}_{\mathrm{BM}}$, the RS provides an additional transmission channel, which is much better than the direct link between BS and MS, and thus greatly improves the capacity.

The capacity gains of the four DF relaying modes are plotted in Fig. 8, and the maximum value of the four gains can be seen as the gain of the proposed DF relaying scheme. It is shown that the proposed DF relaying scheme always provides a positive capacity gain. With $\mathrm{SNR}_{\mathrm{MR}}$ increasing in Fig. 8a, the capacity gain of BDF also 

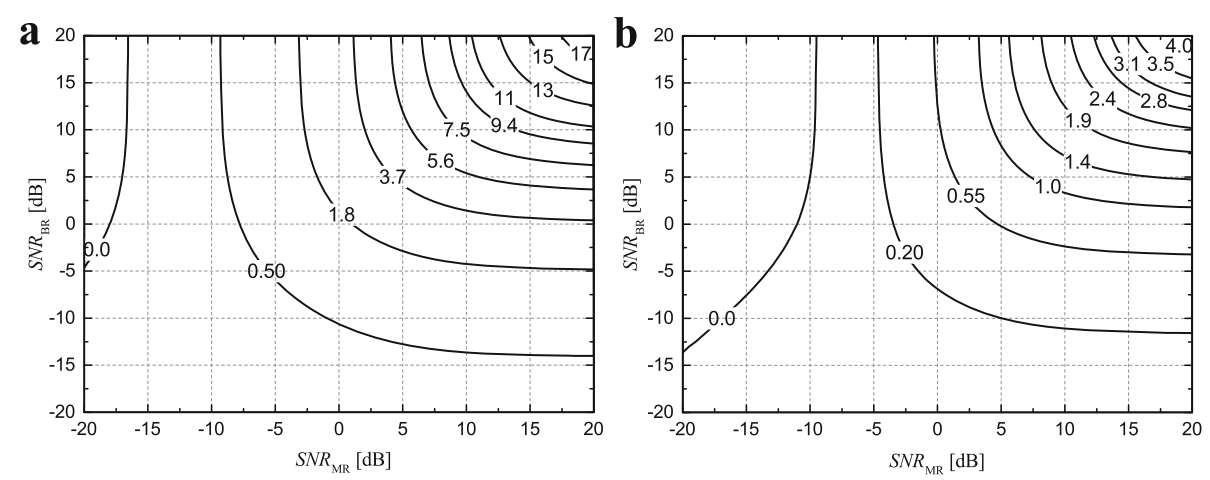

Fig. 7 Contour plots of capacity gain in $A F$ relaying scheme for $\mathbf{a} S N R_{B M}=-10 \mathrm{~dB}$ and $\mathbf{b} S N R_{B M}=5 \mathrm{~dB}$

increases, while the capacity gain of DDF keeps constant value for large $\mathrm{SNR}_{M R}$. This is because for larger $\mathrm{SNR}_{M R}$, the sum-capacity of DDF mode is limited by the upper bound of (22) at the first phase, which is not affected by the $\mathrm{SNR}_{\mathrm{MR}}$ for severe interference. It is also shown that for the region around $\mathrm{SNR}_{\mathrm{MR}}=5 \mathrm{~dB}$, the capacities of the BDF and DDF are the same. In this case, the relay network with the DDF mode suffers from a very strong interference, and the sum-capacities of BDF and DDF are all upper bounded by the sum-rate at the first phase as shown in Figs. 4d and 6c. In Fig. 8b, it is shown that the capacity gain of $\mathrm{BDF}$ is always larger than the other modes. For $\mathrm{SNR}_{\mathrm{MR}}=10 \mathrm{~dB}$ and $\mathrm{SNR}_{\mathrm{BR}}$ is lower than $15 \mathrm{~dB}$, the DDF mode suffers from a very strong interference at the first phase. Moreover, it is also shown that with $\mathrm{SNR}_{\mathrm{BR}}$ increasing, the capacity gain of UDF first increases and then decreases. The maximum point is achieved when the uplink rate at the first phase equals to that of the second phase. For small value of SNR $\mathrm{BR}_{\mathrm{BR}}$, UDF suffers a weak downlink interference at the first phase, and the sum- capacity is mainly limited by the rates at the second phase. With $\mathrm{SNR}_{\mathrm{BR}}$ increasing, the sum-capacity is limited by the strong interference at the first phase, and the capacity gain becomes a decreasing function of $\mathrm{SNR}_{\mathrm{BR}}$.

The best mode selected by the proposed DF relaying scheme is shown in Fig. 9 for a fixed value of SNR $\mathrm{BR}_{\text {. Sim- }}$ ulation results verify the analysis in the above section, i.e., if $\mathrm{SNR}_{\mathrm{MR}}$ is much higher than others, the BDF mode is the best, and if $\mathrm{SNR}_{\mathrm{BM}}$ is much larger than $\mathrm{SNR}_{M R}$ and $\mathrm{SNR}_{\mathrm{BR}}$, the best mode is UDF; if $\mathrm{SNR}_{\mathrm{BM}}$ is much larger than both the $S_{N R}$ MR and SNR $R_{B R}$, NF is the best; if $S_{N R}$ is much smaller than $\mathrm{SNR}_{\mathrm{BM}}$ and $\mathrm{SNR}_{\mathrm{BR}}$, and at the same time $\mathrm{SNR}_{\mathrm{BR}}$ is smaller than $\mathrm{SNR}_{\mathrm{BM}}$, the DDF mode is the best. Note that the non-symmetry of the UDF and DDF mode is caused by the fact that the BS operates in FD mode, while the MS operates in HD mode, and in addition, the non-symmetrical of uplink and downlink channel conditions.

The sum-capacities of the proposed AF and DF relaying schemes are shown in Figs. 10 and 11, which are plotted
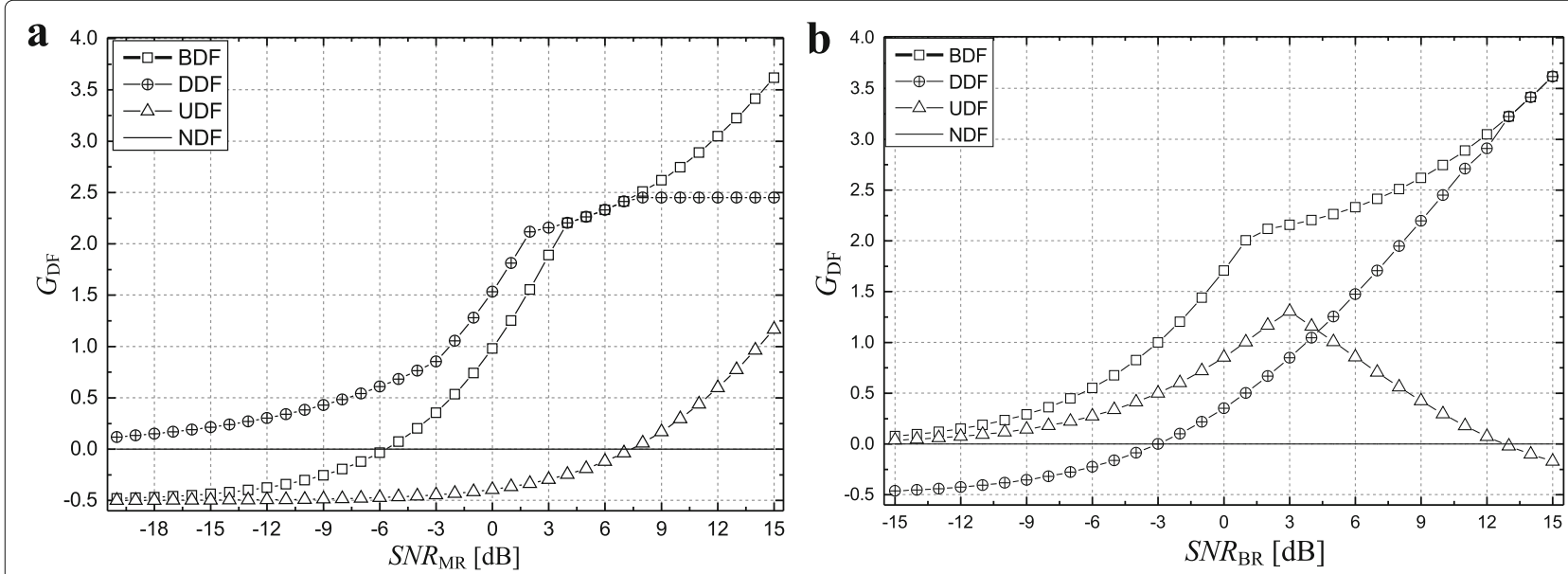

Fig. 8 The comparisons among the capacity gains of four DF modes in two typical cases. a SNRBM $=-3 \mathrm{~dB}, \mathrm{SNR} R_{B R}=10 \mathrm{~dB}$. $\mathbf{b} S N R_{B M}=-3 \mathrm{~dB}$, $S N R_{M R}=10 \mathrm{~dB}$ 

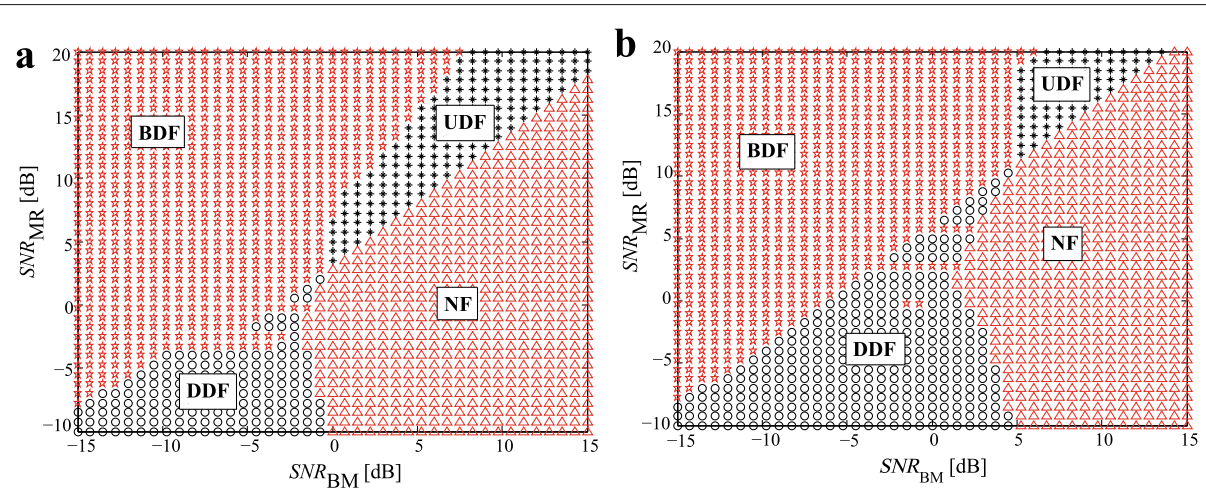

Fig. 9 The mode selection regions for $\mathbf{a} S N R_{B R}=0 \mathrm{~dB}$ and $\mathbf{b} S N R_{B R}=7 \mathrm{~dB}$

for $S_{N R} R_{B M}=-3 \mathrm{~dB}$ as functions of $S N R_{B R}$ and $S N R_{M R}$, respectively. It is shown that when $S N R_{B R}$ and $S N R_{M R}$ are very small, the sum-capacity of the AF relaying scheme is larger than that of the DF relaying scheme. This is because to guarantee error-free decoding at RS requires very low transmission rates when the channels from the BS and MS to RS experience deep fading. The best relaying mode selected by the proposed DF scheme is the NF mode. In fact, in this case, the RS cannot decode the information bits from BS and MS correctly, due to its receive SNR which is too small. However, in the AF relaying scheme, the RS only amplifies and forwards the signal, and thus increases the SNRs at the receivers of the BS and MS. However, as $S N R_{B R}$ and $S N R_{M R}$ increase, the DF relaying scheme outperforms the AF relaying scheme. This is because in the AF relaying scheme, the received noise is also amplified and forwarded with the signal; however, in the DF relaying scheme, the RS can obtain a noisefree message by decoding the received signal, and then forwards a re-encoded signal.

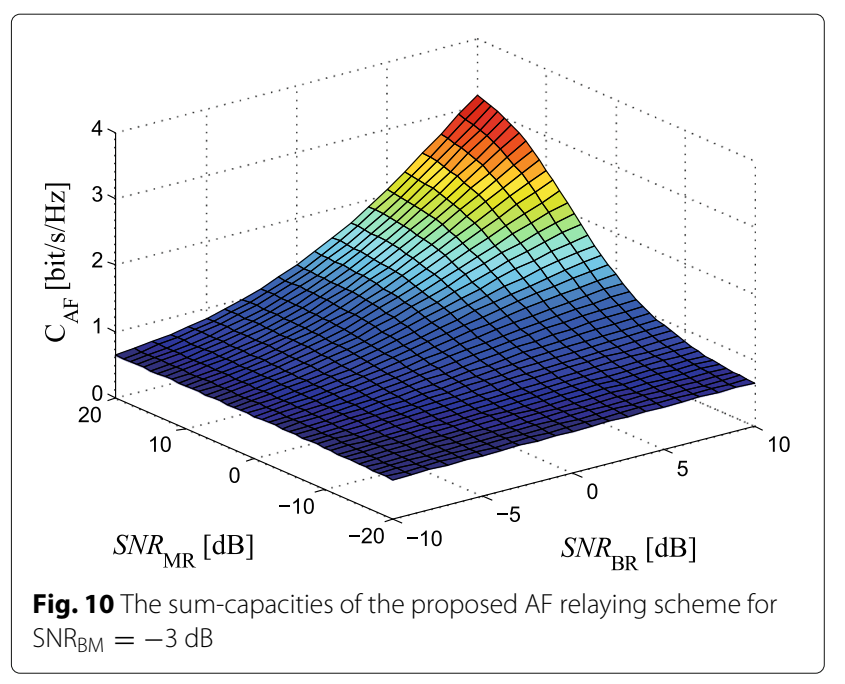

Finally, we show the performance of the proposed DF relaying modes in a cellular system. The BS antenna is a $120^{\circ}$ sector antenna located in the center of the cell. The geometry of the sector is presented in the rectangular coordinate system with $x$ - and $y$-axes. The radius of the cell is $876 \mathrm{~m}$, and the RS is $526 \mathrm{~m}$ away from the BS. The transmit power is $P=23 \mathrm{dBm}$ and the receive noise power is $\sigma^{2}=-144 \mathrm{dBw}$ for all nodes. We assume that the channel experiences a large-scale path loss, which is proportional to $d^{\alpha}$, where $d$ is the propagation distance and $\alpha=3.5$ is the path loss factor.

As shown in Fig. 12, the four modes dominate others in different regions. Due to that the positions of the BS and $\mathrm{RS}$ are fixed, $\mathrm{SNR}_{\mathrm{BR}}$ is equal to about $10.3 \mathrm{~dB}$. When the MS is far away from the BS, and close to the RS, the RS can effectively enhance the capacity for both uplink and downlink, thus the BDF mode is the best. When the MS is close to the BS but far away from the RS, the RS is useless for improving the sum-capacity, the NF mode is the best. When the MS is far away from the RS, and

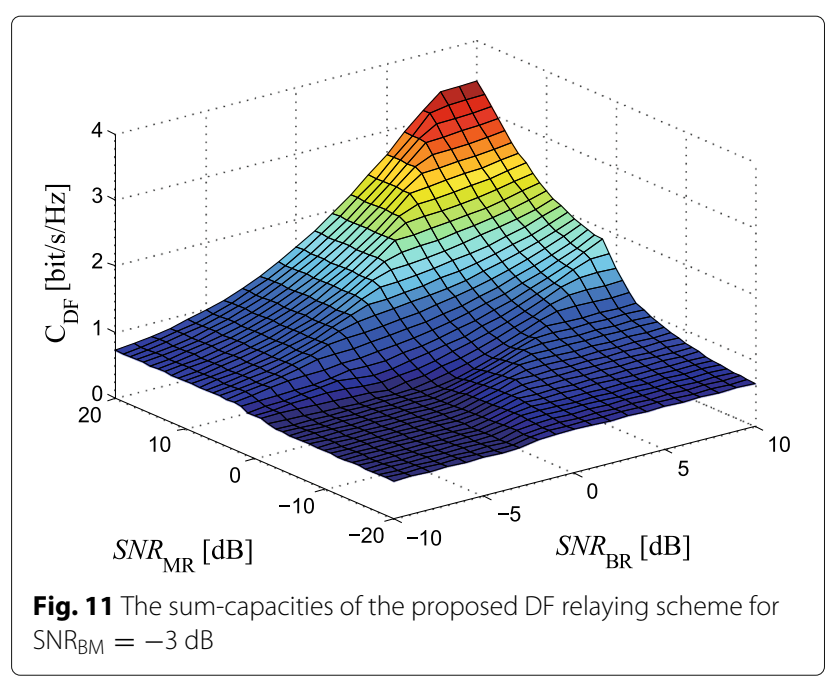




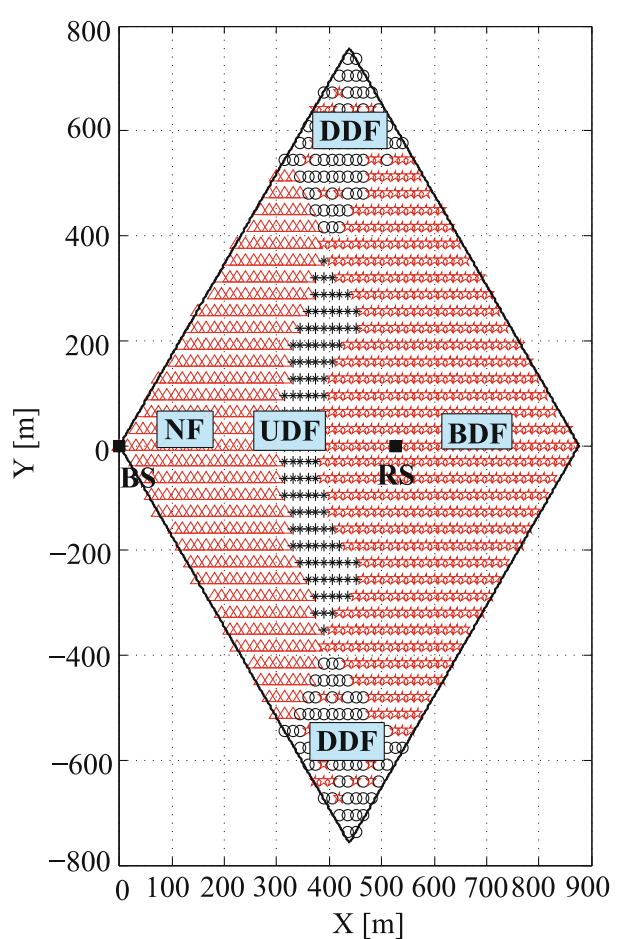

Fig. 12 The mode selection areas in the $120^{\circ}$ sector by adopting the hybrid DF relaying scheme

the distance from the BS to RS is almost equal to the distance from the BS to MS, the optimal gain can be obtained by employing the DDF mode. When the MS is located between the BS and RS, the UDF mode can be applied to achieve higher capacity. Correspondingly, Fig. 13 shows the capacity gain in the sector to measure the performance advantages of the hybrid DF relaying scheme. It is shown that by using the proposed DF relaying scheme, the capacity performance is greatly improved for the users at the cell edge.

\section{Conclusions}

We have studied a three-node relay network, where a half duplex RS assists a full duplex BS to communicate with a half duplex MS. To fully exploit the advantages of FD and relay techniques, two novel relaying schemes are proposed, one for AF relaying and another for DF relaying. The former one is derived from the traditional two-way relay AF relaying scheme by just enabling the BS to transmit and receive at the two phases. The later one is designed to achieve the maximum sum-capacity by choosing the best relaying mode from four modes. The achievable rate region and sum-capacity of each mode are also derived in closed form. Simulation results show that the proposed schemes provide dramatic capacity gain. In addition, for the proposed DF relaying scheme, the best

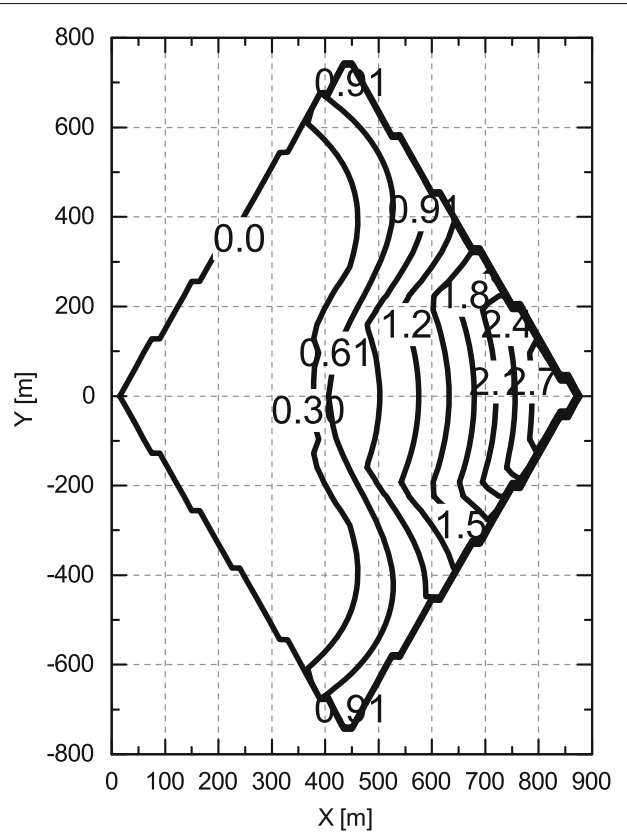

Fig. 13 Contour plots for the overall sum-capacity gains offered by the hybrid DF relaying scheme in the $120^{\circ}$ sector

mode are analyzed for different channel conditions. As it is shown in the results, the four modes dominate others in different regions.

\section{Acknowledgments}

This work was jointly supported by the National Natural Science Foundation of China under Grant No. 61671024, and the Hong-Kong, Macao and Taiwan Science \& Technology Cooperation Program of China under Grant No. 2016YFE0123200.

\section{Competing interests}

The authors declare that they have no competing interests.

Received: 5 September 2016 Accepted: 9 February 2017

Published online: 04 March 2017

\section{References}

1. Jl Choi, K Srinivasan, M Jain, P Levis, S Katti, in Proc. ACM MobiCom. Achieving single channel, full duplex wireless communication (ACM, Chicago, 2010), pp. 1-12

2. D Bharadia, E McMilin, S Katti, Full duplex radios. Proc. ACM SIGCOMM. 43(4), 375-386 (2013)

3. Y Hua, P Liang, Y Ma, AC Cirik, Q Gao, A method for broadband full-duplex MIMO radio. IEEE Signal Process. Lett. 19(12), 793-796 (2012)

4. H Ju, E Oh, D Hong, Improving efficiency of resource usage in two-hop full duplex relay systems based on resource sharing and interference cancellation. IEEE Trans. Wireless Commun. 8(8), 3933-3938 (2009)

5. E Everett, A Sahai, A Sabharwal, Passive self-interference suppression for full-duplex infrastructure nodes. IEEE Trans. Wireless Commun. 13(2), 680-694 (2014)

6. M Duarte, C Dick, A Sabharwal, Experiment-driven characterization of full-duplex wireless systems. IEEE Trans. Wireless Commun. 11(12), 4296-4307 (2012)

7. TRiihonen, S Werner, R Wichman, Mitigation of loopback self-interference in full-duplex MIMO relays. IEEE Trans. Signal Process. 59(12), 5983-5993 (2011) 
8. NH Mahmood, G Berardinelli, FML Tavares, P Mogensen, in Proc. IEEE 81st Vehicular Technology Conference: VTC-Spring. On the potential of full duplex communication in $5 \mathrm{G}$ small cell networks, (Glasgow, 2015), pp. 1-5

9. A Thangaraj, RK Ganti, S Bhashyam, in Proc. Int. Conf. Signal Process. Commun. Self-interference cancellation models for full-duplex wireless communications (SPCOM, Bangalore, 2012), pp. 1-5

10. Z Zhang, X Chai, K Long, AV Vasilakos, L Hanzo, Full duplex techniques for $5 \mathrm{G}$ networks: self-interference cancellation, protocol design, and relay selection. IEEE Commun. Mag. 53(5), 128-137 (2015)

11. Z Wang, L Li, H Wang, H Tian, Beamforming design in relay-based full-duplex MISO wireless powered communication networks. IEEE Commun. Lett. 20(10), 2047-2050 (2016)

12. $L L u, X Z$ hou, $\cup$ Onunkwo, $G L i$, Ten years of research in spectrum sensing and sharing in cognitive radio. EURASIP J. Wirel. Commun. Netw. 2012(1), 1 (2012)

13. A Sahai, G Patel, C Dick, A Sabharwal, in Proc. Asilomar Conference on Signals, Systems and Computers. Understanding the impact of phase noise on active cancellation in wireless full-duplex, (Pacific Grove, 2012), pp. 29-33

14. A Nosratinia, TE Hunter, A Hedayat, Cooperative communication in wireless networks. IEEE Commun. Mag. 42(10), 74-80 (2004)

15. N Jindal, S Vishwanath, A Goldsmith, On the duality of gaussian multiple-access and broadcast channels. IEEE Trans. Inf. Theory. 50(5), 768-783 (2004)

16. A Sheikh, A Olfat, New beamforming and relay selection for two-way decode-and-forward relay networks. IEEE Trans. Veh. Technol. 65(3), 1354-1366 (2016)

17. JN Laneman, DNC Tse, GW Wornell, Cooperative diversity in wireless networks: efficient protocols and outage behavior. IEEE Trans. Inf. Theory. 50(12), 3062-3080 (2004)

18. F Zhao, X Sun, H Chen, R Bie, Outage performance of relay-assisted primary and secondary transmissions in cognitive relay networks. EURASIP J. Wirel. Commun. Netw. 1, 1-10 (2014)

19. X Jia, X Dang, M Zhou, L Yang, H Zhu, Adaptive power allocation and outage performance of cognitive best relay cooperation systems with multiple primary transceiver pairs and direct path between cognitive source and destination. EURASIP J. Wirel. Commun. Netw. 2014(1), 1-14 (2014)

20. A Lo, P Guan, in Proc. 2011 Int. Conf. Inform. Networking. Performance of in-band full-duplex amplify-and-forward and decode-and-forward relays with spatial diversity for next-generation wireless broadband, (Kuala Lumpur, 2011), pp. 290-294

21. TRiihonen, S Werner, R Wichman, in Proc. Wireless Commun. and Networking Conf. Comparison of full-duplex and half-duplex modes with a fixed amplify-and-forward relay, (Budapest, 2009), pp. 1-5

22. T Liu, C Yang, Equivalent signal-alignment-based frequency-domain equalization for MC-CDMA two-way relay systems. IEEE Trans. Veh. Technol. 61(1), 237-248 (2012)

23. H Ju, E Oh, D Hong, Catching resource-devouring worms in next-generation wireless relay systems: two-way relay and full-duplex relay. IEEE Commun. Mag. 47(9), 58-65 (2009)

24. Z Zhang, Z Ma, Z Ding, M Xiao, GK Karagiannidis, Full-duplex two-way and one-way relaying: average rate, outage probability, and tradeoffs. IEEE Trans. Wireless Commun. 15(6), 3920-3933 (2016)

25. J Lee, M Rim, K Kim, Availability of direct path in half-duplex-based cooperative relay networks. EURASIP J. Wirel. Commun. Netw. 2015(1), $1-15(2015)$

26. K Woradit, TQS Quek, W Suwansantisuk, H Wymeersch, L Wuttisittikulkij, MZ Win, Outage behavior of selective relaying schemes. IEEE Trans. Wireless Commun. 8(8), 3890-3895 (2009)

27. A Bletsas, H Shin, MZ Win, Cooperative communications with outage-optimal opportunistic relaying. IEEE Trans. Wireless Commun. 6(9), 3450-3460 (2007)

28. A Host-Madsen, Capacity bounds for cooperative diversity. IEEE Trans. Inf. Theory. 52(4), 1522-1544 (2006)

29. T Riihonen, S Werner, R Wichman, Hybrid full-duplex/half-duplex relaying with transmit power adaptation. IEEE Trans. Wireless Commun. 10(9), 3074-3085 (2011)

30. M Chraiti, W Ajib, JF Frigon, in Proc. IEEE Global Commun. Conf. Distributed Alamouti full-duplex relaying scheme with direct link, (Atlanta, 2013), pp. 4020-4025
31. T Kwon, S Lim, S Choi, D Hong, Optimal duplex mode for DF relay in terms of the outage probability. IEEE Trans. Veh. Technol. 59(7), 3628-3634 (2010)

32. B Rankov, A Wittneben, in Proc. IEEE Int. Symp. Inf. Theory. Achievable rate regions for the two-way relay channel, (Seattle, 2006), pp. 1668-1672

33. H Cui, M Ma, L Song, B Jiao, Relay selection for two-way full duplex relay networks with amplify-and-forward protocol. IEEE Trans. Wireless Commun. 13(7), 3768-3777 (2014)

34. S Luo, P Liu, S Panwar, in Proc. IEEE. Veh. Technol. Conf. Full-duplex relaying in an infrastructure-based wireless network, (Vancouver, 2014), pp. 1-6

35. L Pinals, M Vu, Link-state optimized decode-forward transmission for two-way relaying. IEEE Trans. Commun. 64(5), 1844-1860 (2016)

36. B Rankov, A Wittneben, Spectral efficient protocols for half-duplex fading relay channels. IEEE. J. Sel. Areas Commun. 25(2), 379-389 (2007)

37. P Popovski, H Yomo, in Proc. Int. Conf. Commun. Physical network coding in two-way wireless relay channels, (Glasgow, 2007), pp. 707-712

38. H Rasouli, A Anpalagan, in Proc. 25th QBSC. SNR-based vs. BER-based power allocation for an amplify-and-forward single-relay wireless system with MRC at destination, (Kingston, 2010), pp. 429-432

39. M Vu, MISO capacity with per-antenna power constraint. IEEE Trans. Commun. 59(5), 1268-1274 (2011)

40. D Tse, P Viswanath, in Fundamentals of wireless communication (Cambridge University Press, UK, 2005), pp. 228-289

41. A Goldsmith, Wireless communications. (Cambridge University Press, UK, 2005)

42. SJ Kim, P Mitran, V Tarokh, Performance bounds for bidirectional coded cooperation protocols. IEEE Trans. Inf. Theory. 54(11), 5235-5241 (2008)

43. AEl Gamal, YH Kim, Network information theory. (Cambridge University Press, UK, 2011), pp. 151-158

44. M Costa, On the Gaussian interference channel. IEEE Trans. Inf. Theory. 31(5), 607-615 (1985)

45. I Sason, in Proceedings. International Symposium on Information Theory (IIIT). On achievable rate regions for the gaussian interference channel, (Chicago, 2004), p. 1

46. G Kramer, Outer bounds on the capacity of gaussian interference channels. IEEE Trans. Inf. Theory. 50(3), 581-586 (2004)

\section{Submit your manuscript to a SpringerOpen ${ }^{\circ}$ journal and benefit from:}

- Convenient online submission

- Rigorous peer review

- Immediate publication on acceptance

- Open access: articles freely available online

- High visibility within the field

- Retaining the copyright to your article

Submit your next manuscript at $>$ springeropen.com 